\title{
HIGHLIGHTS OF THE ISO LONG-WAVELENGTH SPECTROMETER
}

\author{
PETER E. CLEGG \\ Queen Mary and Westfield College \\ Mile End Road, London E1 4 NS
}

\section{Introduction}

It is a pleasure for me to present, on behalf of the international consortium of scientist and engineers who built the instrument, some interim results from the Long-Wavelength Spectrometer (LWS). I shall briefly review the scientific capabilities of the instrument before describing some of the highlights of its scientific programme.

\section{Description and Performance of the Instrument}

Figure 1 shows the interior of the LWS. The instrument consists of a grating spectrometer covering the wavelength range $43-196.9 \mu \mathrm{m}$, the lower end of this range overlapping with that of the Short-Wavelength Spectrometer (de Grauuw, this volume). The spectral resolution element in this mode is $0.29 \mu \mathrm{m}$ between $43 \mu \mathrm{m}$ and $94.6 \mu \mathrm{m}$ and $0.60 \mu \mathrm{m}$ between $94.6 \mu \mathrm{m}$ and $196.9 \mu \mathrm{m}$. The spectral resolving power can be increased by moving one of two Fabry-Perot etalons into the beam of radiation. In this mode, the resolving power varies between 8100 and 8500 , for the wavelength range $47-70 \mu \mathrm{m}$, and between 6800 and 9700 for the wavelength range $70-196.6 \mu \mathrm{m}$; these figures correspond to a velocity resolution of between 31 and $44 \mathrm{~km} \mathrm{~s}^{-1}$. Like ISOCAM (Cesarsky, this volume) and ISOPHOT (Lemke, this volume), the LWS can be operated at reduced data-rate when another instrument is being used. This so-called "serendipitously-parallel" mode provides narrowband photometry at 10 fixed wavelengths.

The performance of the instrument in orbit is, on the whole, very similar to ground-based expectation. The effects of particle hits have proved more significant than the ground-based tests had suggested, reducing the sensitivity, and the spectra of extended sources exhibit channel fringes, which can be removed during ground processing. In spite of the reduced sensitivity, our programme of observations has had to be modified only in detail and we have still been able to pursue our main objectives. The calibration of the instrument is already well within the pre-launch limits of $\pm 30 \%$ absolute and continues to improve.

\section{Scientific Objectives and Achievements}

Our objective before launch was fully to exploit the capabilities of the instrument in exploring the new window opened by ISO. We intended to use previously unattainable lines for diagnostic purposes as well as seeking new lines or solid state features. At the higher resolution offered by the Fabry-Perots, we intended to make kinematical studies as well as for studying weak lines on strong continua. The range of the LWS' astronomical programmes is wide, ranging from the Solar System to ultraluminous galaxies. I do not have space here to give examples from every programme and I restrict myself to some representative examples.

\subsection{THE SOLAR SYSTEM}

A major goal of our programme is the determination of the cosmogonically interesting ratio of deuterium to hydrogen in the atmospheres of the giant planets. Ground-based determinations are difficult, involving the complex and uncertain chemistry of $\mathrm{CH}_{3} \mathrm{D}, \mathrm{CH}_{4}, \mathrm{HD}$ and $\mathrm{H}_{2}$. LWS 


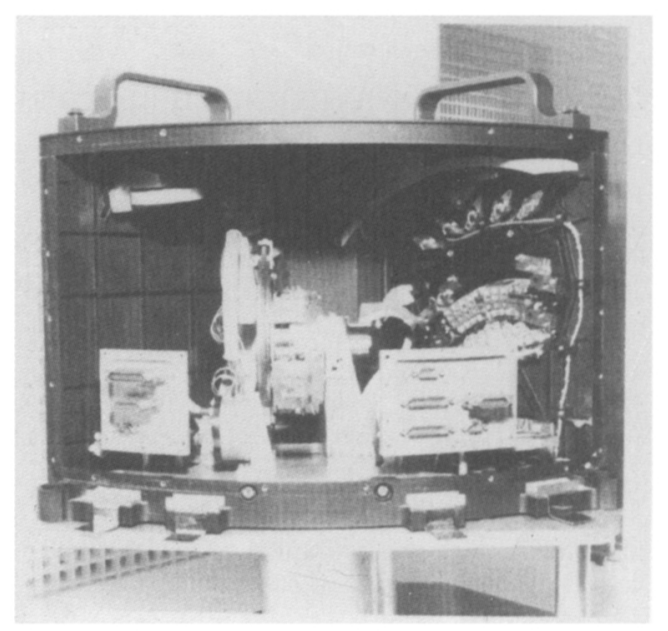

Figure 1. Interior view of the LWS

preliminary results for Saturn (Griffin et al., 1996 and Griffin, private communication) are consistent with the mutually incompatible ground-based figure for Saturn and the in situ measurements of Jupiter. As the atmospheres of these planets were expected to be very similar in this respect, refined LWS figures are expected to throw more light on this discrepancy.

\subsection{WATER VAPOUR}

Before ISO, only maser lines had been seen from the water molecule and, because of the complex exitation mechanism, these cannot be used as reliable diagnostics of the physical conditions in sources. One of the goals of the LWS programme was therefore to look for water vapour in a wide variety of sources; this goal has been abundantly achieved. Figure 2 (Barlow et al., 1996) shows the very rich water vapour emission spectrum of - appropriately - W Hya; CO is also clearly present, as well as $\mathrm{H}_{2} \mathrm{O}$. Water vapour has been detected in a variety of sources in emission and absorption, from the Herbig-Haro object HH54 (Liseau et al., 1996) to lines of sight towards Sgr B2 (Cernicharo et al., 1997).

\subsection{DISCOVERY OF NEW SPECIES}

Naturally, we hoped that the LWS would make discoveries. Neufeld, Zmuidzinas, Schilke and Phillips (1997) have made first detection of an interstellar halogen molecule other than chlorine hydrogen fluoride in Sgr B2. The low concentration of $\mathrm{HF}$ relative to $\mathrm{H}$ suggests strong depletion of the former on to grains. Similarly, Cernicharo et al. (1997) have observed the rotational spectrum of the radical $\mathrm{CH}^{+}$in $\mathrm{NGC7027.}$

\subsection{ULTRALUMINOUS GALAXIES}

Fine-structure lines are very important diagnostic tools that we have used to investigate the conditions in many sources. To our surprise, we found that the ultraluminous galaxy Arp 220 shows only very weak lines, particularly [CII] $(158 \mu \mathrm{m})$. This is in contrast to less luminous galaxies such as Arp 229 (Luhman et al., 1997), which is in an earlier stage of interaction and which shows copious fine-structure lines. Although several explanations for this have been advanced, we are making more observations to try to elucidate the problem. 

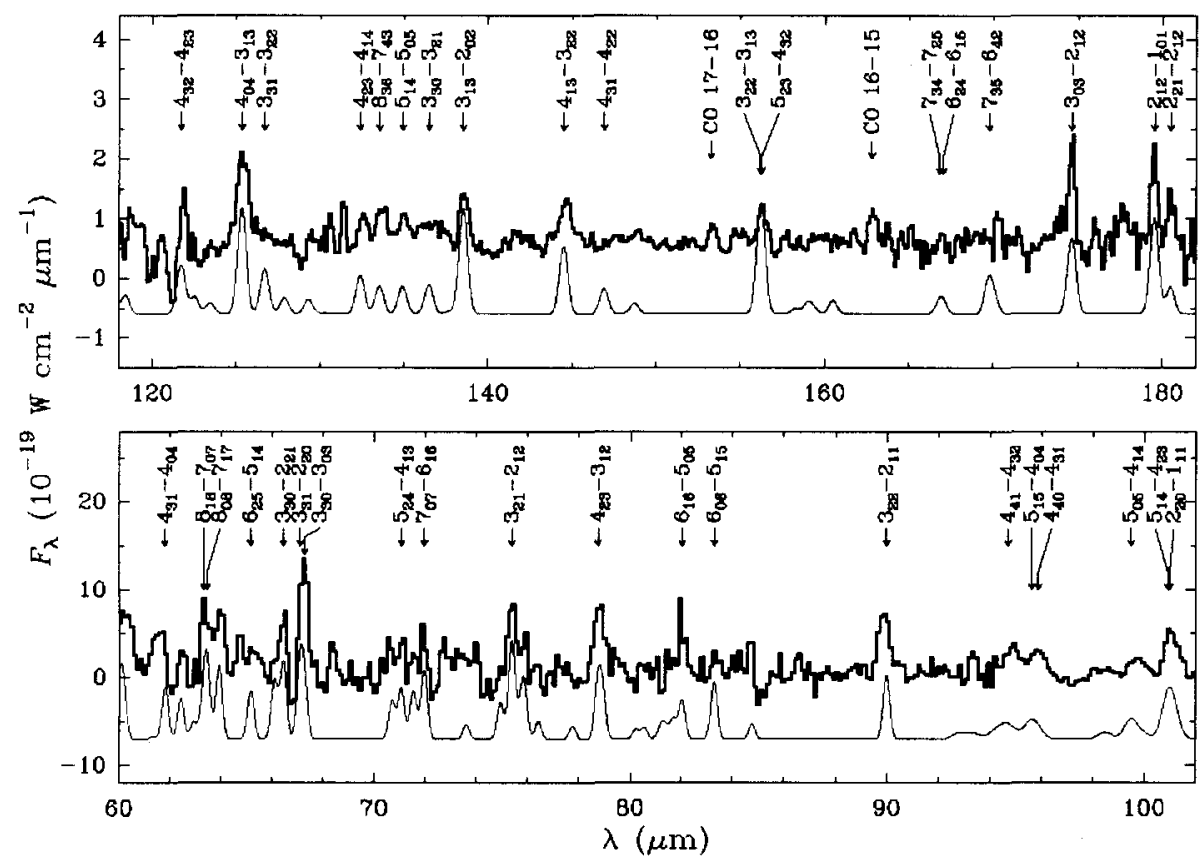

Figure 2. The spectrum of $\mathrm{W}$ Hya together with a model fit to the water vapour lines

\subsection{SERENDIPITOUSLY-PARALLEL MODE}

Although conceived well after the design of the LWS, the serendipitously-parallel mode is proving extremely valuable. Maps of the galactic centre region made in parallel with ISOCAM raster maps, for example, whilst in excellent agreement with IRAS maps at the two corresponding wavelength, show finer spatial detail extend to longer wavelengths and provide more spectrophotometric information (Caux, private communication).

\section{Conclusion}

ISO has been in orbit for some twenty-four months and the results have already more than justified the mission. Yet the mission is expected to continue for another four months or so and the instruments continue to deliver a plethora of data.

\section{Acknowlegements}

I am indebted to the LWS Consortium, for their efforts in designing and building the LWS instrument, and to ESA for providing and operating the outstanding ISO telescope and satellite.

\section{References}

Barlow, M. J., Ngyuen-Q-Rieu, Troung-Bach et al., 1996, A\&A, 315, L241

Cernicharo, J., Lim, T., Cox, P., González-Alfonso, E., Caux, E., Swinyard, B. M., Martín-Pintado, J., Baluteau, J. P., Clegg, P., 1997, A\&A, 323(1), L25

Cernicharo, J., Liu, X.-W., Cox, P., Barlow, M. J., Lim, T., Swinyard, B. M., 1997, ApJ, 483, L65

Fischer, J., Satyapal, S., Luhman, M. L. et al., 1997, to appear in Mamon, Trinh Xuân Thuân and Trân, Thanh Vân (eds.) "Extragalactic Astronomy in the Infrared", Editions Frontiéres.

Griffin, M. J., Naylor, D. A., Davis, G. R. et al., 1996, A\&A, 315, L3891996, A\&A, 315, L241

Liseau, R., Ceccarelli, C., Larson, B. et al., 1996, A\&A, 315, L181

Luhman, M. L., Fischer, J, Satyapal, S. et al., 1997, to appear in Mamon, Trinh Xuân Thuân and Trân, Thanh Vân (eds.) "Extragalactic Astronomy in the Infrared", Editions Frontiéres.

Neufeld, D. A., Zmuidzinas, J., Schilke, P., Phillips, T. G., 1997, ApJ, 488, L1411997 